\title{
Dutch food bank parcels do not meet nutritional guidelines for a healthy diet
}

\author{
Judith E. Neter ${ }^{1 *}$, S. Coosje Dijkstra ${ }^{1}$, Marjolein Visser ${ }^{1,2}$ and Ingeborg A. Brouwer ${ }^{1}$ \\ ${ }^{1}$ Department of Health Sciences, The EMGO Institute for Health and Care Research, Faculty of Earth and Life Sciences, \\ VU University Amsterdam, De Boelelaan 1085, $1081 \mathrm{HV}$ Amsterdam, The Netherlands \\ ${ }^{2}$ Department of Nutrition and Dietetics, Internal Medicine, VU University Medical Center, De Boelelaan 1117,1081 HV \\ Amsterdam, The Netherlands
}

(Submitted 8 October 2015 - Final revision received 22 March 2016 - Accepted 27 April 2016 - First published online 27 May 2016)

\section{Abstract}

Nutritional intakes of food bank recipients and consequently their health status largely rely on the availability and quality of donated food in provided food parcels. In this cross-sectional study, the nutritional quality of ninety-six individual food parcels was assessed and compared with the Dutch nutritional guidelines for a healthy diet. Furthermore, we assessed how food bank recipients use the contents of the food parcel. Therefore, 251 Dutch food bank recipients from eleven food banks throughout the Netherlands filled out a general questionnaire. The provided amounts of energy (19849 (sD 162 615) kJ (4744 (sD 38 866) kcal)), protein (14.6 energy percentages (en\%)) and SFA (12.9 en\%) in a single-person food parcel for one single day were higher than the nutritional guidelines, whereas the provided amounts of fruits (97 (SD 1441) g) and fish (23 (sD 640) g) were lower. The number of days for which macronutrients, fruits, vegetables and fish were provided for a single-person food parcel ranged from 1.2 (fruits) to 11.3 (protein) d. Of the participants, only $9.5 \%$ bought fruits and $4.6 \%$ bought fish to supplement the food parcel, $39.4 \%$ used all foods provided and $75.7 \%$ were (very) satisfied with the contents of the food parcel. Our study shows that the nutritional content of food parcels provided by Dutch food banks is not in line with the nutritional guidelines. Improving the quality of the parcels is likely to positively impact the dietary intake of this vulnerable population subgroup.

\section{Key words: Nutrition guidelines: Food parcels: Public health issues: Food banks: Socio-economic status}

Even in wealthy Western countries such as the Netherlands, food banks are needed to help people who cannot make ends meet. The Dutch Food Bank is a charitable, non-governmental organisation that collects and distributes donated foods through 160 food banks with 510 distribution points throughout the Netherlands. In 2014, the food banks weekly provided over 37000 food parcels, and thereby supported approximately 94000 individuals (5\% of the poorest households in the Netherlands), which is $5 \cdot 3$ times higher compared with $2006^{(1)}$.

The Dutch Food Bank aims to weekly provide food parcels that supplement the normal diet for $2-3 \mathrm{~d}$. The contents of the food parcels largely depend on donated foods by food companies, supermarkets and individuals, and consequently varies by week and by food bank. Foods donated by food companies include products with damaged packages, products nearing their expiration date, products that cannot be stored due to storage shortage and products with manufacturing errors such as mislabelling. Foods donated by supermarkets are mainly products nearing their expiration date. Individuals mainly donate non-perishable foods from shopping lists supplied by the Dutch Food Bank.

Food bank recipients are a group of concern as they have a very low socio-economic status (SES) and have limited resources to purchase food. Therefore, they largely rely on the availability and quality of donated food in the food parcels. We have previously shown that $72.9 \%$ of the Dutch food bank recipients are food insecure, of which $40.4 \%$ have very low food security ${ }^{(2)}$. Food insecurity can be defined as the lack of availability of nutritionally adequate and safe foods, or the lack of ability to acquire acceptable foods in socially acceptable ways $^{(3)}$. Both low SES and food insecurity have been associated with a less-healthy diet. Food insecure people have a lower intake of fruits and vegetables ${ }^{(4-9)}$ and a lower nutrient intake $^{(6,8-12)}$, which may lead to micronutrient deficiencies and malnutrition $^{(11,13)}$. Furthermore, food insecurity has been shown to be associated with poorer health including poor oral health $^{(14)}$, overweight, diabetes and heart disease, and consequently is a major public health issue ${ }^{(15-20)}$. Food intake of food bank recipients is assumed to be unhealthy with consequently a higher risk for diseases. Therefore, it is of great importance that the nutritional quality of the food parcels is optimal and that food bank recipients can meet the nutritional guidelines.

It is unknown whether the quality of food parcels is sufficient to contribute to a healthy diet and how food bank recipients use the contents of the food parcels. Therefore, the aims of this study were to assess the nutritional content of food parcels

Abbreviations: IQR, interquartile ranges; NEVO-code, Dutch Food Composition Table code.

* Corresponding author: J. E. Neter, fax +31 205986 940, email judith.neter@vu.nl 
provided by Dutch food banks, to compare this with the Dutch nutritional guidelines for a healthy diet ${ }^{(21-23)}$ and to assess how food bank recipients use the contents of their food parcels.

\section{Methods}

This cross-sectional study was a part of the Dutch Food Bank study, which aimed to explore and optimise food choices and food patterns among Dutch food bank recipients. The study was approved by the Medical Ethics Committee of the VU Medical Center in Amsterdam, The Netherlands, as well as the national board of the Dutch Food Bank.

Out of 135 (approximately) Dutch food banks, eleven were selected for the present study. The inclusion criterion for food banks to participate was provision of food parcels once a week. Furthermore, the selection of food banks was on the basis of the number of recipients (smaller $(n<50)$, medium $(n=50-100)$ as well as larger $(n>100)$ numbers of recipients), urbanisation level (smaller (e.g. 30000 inhabitants) as well as larger (e.g. >120000 inhabitants) cities), region (different regions across the Netherlands) and willingness to participate in this study. The participating food banks were located in Apeldoorn, Boxtel, Breda, Enschede, Groningen, Haarlem, Hilversum, Huizen, Rotterdam, Wageningen and Zeewolde.

\section{Food parcels}

The Dutch Food Bank, the national umbrella organisation, aims to provide food parcels that supplement the normal diet for $2-3 \mathrm{~d}$ to those in need. Food banks do not have much influence on the contents of food parcels as the contents largely depend on the availability of donated foods. Therefore, the contents of the food parcels are not based on consumers' taste. Although individual food banks strive to provide food parcels that are as diversified and healthy as possible, food parcels were not composed according to any guideline, for example, regarding nutrient composition or number of products. Furthermore, individual food banks aim to supply food parcels of which the contents are - more or less - the same, by equally dividing all donated foods over the food parcels. The number of foods per food group is predetermined and in case of various sizes of food parcels adapted to the number of people the food parcel is intended for. However, within a food group foods can vary because of the following reasons: (1) in some cases food bank recipients can choose between, for example, white or brown bread, broccoli or tomatoes, or peanut butter or jam and (2) there is not enough of the same food for all recipients. In the first case, we let the volunteers choose for us, we had to make the choices ourselves or we copied the contents of the food parcel of a random food bank recipient.

\section{Nutritional quality of food parcels}

To measure the nutritional quality of food parcels, trained researchers used a standard scoring form, which consisted of general information on the food parcel and information on the foods provided. General information included the date, location of the food bank, type of food parcel (e.g. one to four person household, small, vegetarian) and the number of people the food parcel was intended for. Information on the foods provided in the food parcel included a detailed description (e.g. Vifit strawberry flavoured yogurt drink, light), brand name, number of foods (e.g. two cartons), amount in grams or millilitres (e.g. cartons of $500 \mathrm{ml}$ each) and additional information (e.g. total fat $0.8 \mathrm{~g} / 100 \mathrm{ml}^{(24)}$ ). Between individual food banks, food parcels were distributed in various sizes (one standard parcel for all household sizes $v$. a parcel per household size (e.g. one to nine persons) $v$. different sizes (e.g. small, medium, large)). Therefore, per food bank, various sizes of food parcels were selected to score. Most types (83\%) of food parcels were scored in at least two different weeks to take the variability of the contents into account. At each food bank, food parcels intended for one person, for two persons and for four persons were selected to score. If recipients from different household sizes than one, two or four persons participated in our study, we also scored these sizes of food parcels. In total, information on the contents of ninety-six food parcels was collected between October 2010 and April 2011.

\section{Data entry of food parcels}

Collected data were coded and entered according to a standardised procedure described below. All recorded foods were coded with the corresponding Dutch Food Composition Table code (NEVO code), using the Measures, Weights and Codes 2003 guide (MWC guide) ${ }^{(25)}$. If a product was not in the MWC guide, the product was searched for in the digital version of the NEVO $2010^{(24)}$. If the product was not in the digital version of the NEVO 2010 as well, the best alternative product was searched for in the MWC guide or the digital version of the NEVO 2010, based on the macronutrient composition of the product. These products were documented with their best alternative product and corresponding NEVO code. After coding all products, data were entered in a developed entry screen using the programme Blaise version 4.8 (Statistics Netherlands), which was linked to the latest version of the digital version of the NEVO from 2010. Weights of the individual foods were adjusted for waste due to cleaning, removable and inedible parts, shrinkage and drained weight to enter the most accurate edible contents of the food parcel.

\section{Food parcel recipients}

During the same time period as the contents of the food parcels were scored, we recruited food bank recipients through promotional posters and information letters at eleven food banks to participate in our study. The target population consisted of recipients of the eleven selected Dutch food banks. Inclusion criteria for participation were as follows: (1) $\geq 18$ years of age, (2) sufficiently fluent in Dutch to participate in oral and written interviews, (3) recipient of a Dutch food bank $\geq 1$ month and (4) ability to collect food parcels from the food bank. A single member per household was included. Of the approximately 1200 food bank recipients who received an information letter or might have seen our promotional poster at the food bank, 368 signed up, of which 251 (68.2\%) 
participated in the study; participants were from Apeldoorn ( $n$ 29), Boxtel ( $n$ 11), Breda ( $n$ 42), Enschede ( $n 71)$, Groningen ( $n$ 17), Haarlem ( $n$ 6), Hilversum ( $n$ 16), Huizen ( $n$ 14), Rotterdam ( $n$ 28), Wageningen ( $n$ 12) and Zeewolde ( $n$ 5). Participation was voluntary and confidential. Food bank recipients who were sufficiently fluent in Dutch to participate in oral and written interviews but had difficulties in reading or writing were offered help to fill in the questionnaire. Details on the sampling and data collection procedures have been described elsewhere ${ }^{(2)}$.

\section{Questionnaires}

Participants of the Food Bank study completed a selfadministered general questionnaire, which consisted of questions regarding socio-demographics, anthropometry, grocery shopping and the contents of their food parcels. Questions used for this study are given in the online Supplementary material.

Socio-demographics included date of birth, sex, duration of being recipient of a Dutch food bank (0-6 months, 6-12 months and >12 months), household size (number of people in household) and level of education. We created three levels of education: low (less than completed elementary school), medium (elementary school) and high (general intermediate and lower vocational education, university, college, higher vocational, general secondary and intermediate vocational education). Anthropometry included self-reported height and weight. BMI was calculated as self-reported weight (kg) divided by self-reported height $\left(\mathrm{m}^{2}\right)$. BMI cut-off points of the WHO were used to define weight status ${ }^{(26)}$. With regard to the domain grocery shopping, we asked participants 'How much money do you weekly spend on average on foods and drinks to supplement the food parcel?'. This amount of money was divided by the number of persons in the household to create the variable money spent on groceries per person per week. Furthermore, we asked the participants 'To what extent are you responsible for the daily grocery shopping?' (not at all, a small part, half, a large part, completely) and 'From which food groups do you buy foods most often to supplement the contents of the food parcel?'. On the basis of frequency, respondents were asked to indicate a maximum of two food groups from a list of twenty food groups from the $\mathrm{NEVO}^{(27)}$. Alcoholic beverages were not accounted for.

Questions regarding the food parcels included the following: 'How satisfied are you usually with the contents of the food parcel?' (very unsatisfied, unsatisfied, not unsatisfied/not satisfied, satisfied, very satisfied), 'How healthy do you think the contents of the food parcel are in general?' (very unhealthy, unhealthy, not unhealthy/not healthy, healthy, very healthy), 'Do you usually use all foods from the food parcel?' (never, sometimes, always), 'Do you use perishable foods from the food parcel beyond their expiration date?' (never, sometimes, always), 'Do you use non-perishable foods from the food parcel beyond their expiration date?' (never, sometimes, always) and 'Why don't you use some foods from the food parcel?' (because the food is beyond the expiration date, because I don't know how to use the food, because I don't like the food, because complementary ingredients in the food parcel are missing to use the food, because I don't have time to prepare the food, because I don't have a stove and/or cooking utensils, because I don't feel like it, other). Respondents were asked to choose one answer, which was most often applicable.

To measure the food security status of the participants, trained interviewers used a translated version of the six-item US Department of Agriculture (USDA) Household Food Security Survey Scale ${ }^{(28)}$. The original, validated ${ }^{(29)}$ American questionnaire was translated back and forth for this study. Coding was carried out in accordance with the Guide to Measuring Household Food Security ${ }^{(28)}$. Food security status was defined and classified according to the USDA guidelines: score 0 or 1 is food secure; score $2-4$ is low food security; and score 5-6 is very low food security ${ }^{(28)}$.

\section{Data analyses}

Statistical analyses were performed using SPSS statistics for Windows, version 21.0 (IBM Corp.). Descriptive statistics were used to summarise the food parcel and participant characteristics. Continuous variables are presented as mean values and standard deviations, whereas categorical variables are presented as frequencies and relative frequencies.

The contents of all scored food parcels were standardised to a single-person food parcel for one day to be able to compare the contents of the different types of food parcels. The contents were divided by the actual number of people the food parcel was intended for or if not specified the average household size of participating households making use of this specific type of food parcel at that specific food bank. The contents were then divided by 2.5 as the contents of a food parcel are intended for $2-3 \mathrm{~d}$; because of the differences in the total number of recipients per food bank, a weighing factor was used on the basis of the total number of recipients of the specific food bank at the time the data were collected. This makes the results representative for an average food parcel in the Netherlands. Absolute values $(\mathrm{g})$ are presented as mean values and standard deviations, and relative values are presented as energy percentages (en\%).

When reporting the contents of the food parcel in comparison with the Dutch nutritional guidelines ${ }^{(21-23)}$, the macronutrients, fruits, vegetables and fish were compared with the recommendations for adult males and females aged 19-70 years. The absolute amounts of macronutrients, fruits and vegetables in grams were divided by the recommended amounts in the Dutch nutritional guidelines ${ }^{(21-23)}$ to calculate the number of days these amounts were provided for. In case the nutritional guideline is expressed as a range, the absolute amount of nutrients was divided by both the upper and lower daily recommendation to derive the minimum and maximum number of days, respectively.

To be able to compare the provided amounts of energy, fruits and vegetables per food bank, mean values and standard deviations of the provided amounts were calculated per food bank. Individual food groups provided in the food parcels are presented as frequency and relative frequency. The provided amounts per food group in grams are presented as medians and interquartile ranges (IQR). 


\section{Results}

\section{Food parcels}

Table 1 shows the average weighed provided macronutrients, fruits, vegetables and fish for a single-person food parcel for one single day and a comparison with the nutritional guidelines. The provided amounts of energy (19849 (sD 162615$) \mathrm{kJ}$ (4744 (sD 38866$) \mathrm{kcal})$ ), protein $(14.6 \mathrm{en} \%)$ and SFA $(12.9 \mathrm{en} \%)$ were higher than the nutritional guidelines, whereas the provided amounts of fruits (97 (SD 1441)) and fish (23 (SD 640)) were lower than the nutritional guidelines.

The number of days for which the food parcel would provide sufficient nutrients, fruits, vegetables and fish for a single person ranged from 1.2 to $11.3 \mathrm{~d}$ (Fig. 1) and varied between food banks. The contents of Dutch food parcels are intended to supplement the normal diet for $2.5 \mathrm{~d}$. On average, fruits (1.2) and fish (1.7) were provided for $<2.5 \mathrm{~d}$, whereas fibre $(3 \cdot 1-4 \cdot 1)$, vegetables (3.7), protein $(6 \cdot 4-11 \cdot 3)$, fat (total 3.7-10.5; SFA $5 \cdot 7-8 \cdot 1)$, energy $(4 \cdot 4-6 \cdot 2)$ and carbohydrates $(3 \cdot 1-7 \cdot 8)$ were provided for $>2.5 \mathrm{~d}$.

The mean provided amounts of energy, fruits and vegetables per single-person food parcel for one day varied widely between food banks; the provided amount of energy ranged from 13393 (sD 4971) to 37865 (sD 46635) kJ (3201 (sD 1188) to 9050

Table 1. Average weighed provided nutrients, fruits, vegetables and fish from ninety-six food parcels from eleven food banks throughout the Netherlands for a single-person food parcel for one single day compared with the nutritional guidelines

\begin{tabular}{|c|c|c|c|c|}
\hline & $\begin{array}{l}\text { Absolute } \\
\text { mean }(\mathrm{g})^{\star}\end{array}$ & SD & $\begin{array}{c}\text { Relative } \\
\text { mean } \\
(\mathrm{en} \%)^{\star}\end{array}$ & Guidelines† \\
\hline Energy (kJ) & 19849 & 162615 & & $7950-11297 \ddagger$ \\
\hline Energy (kcal) & 4744 & 38866 & - & 1900-2700‡ \\
\hline Carbohydrates & 589 & 5243 & $49 \cdot 2$ & 40-70 (en\%) \\
\hline $\begin{array}{l}\text { Monosaccharides } \\
\text { and disaccharides }\end{array}$ & 237 & 1588 & $20 \cdot 6$ & - \\
\hline Polysaccharides & 352 & 4036 & 28.5 & - \\
\hline Protein & 172 & 1714 & $14 \cdot 6$ & $8-10(\mathrm{en} \%) \xi$ \\
\hline Fat total & 178 & 1365 & $34 \cdot 1$ & 20-40 (en\%)\| \\
\hline MUFA & 63 & 492 & $12 \cdot 0$ & - \\
\hline PUFA & 31 & 260 & 5.9 & 12 (en\%)ף \\
\hline FSA & 68 & 543 & $12 \cdot 9$ & $10(\mathrm{en} \%)^{\star \star}$ \\
\hline Trans-fat & 4 & 49 & 0.7 & $1(\mathrm{en} \%)^{\star *}$ \\
\hline Fibre & 49 & 512 & - & $30-40(\mathrm{~g})+\dagger$ \\
\hline Fruits & 97 & 1441 & - & $200(\mathrm{~g}) \dagger \dagger$ \\
\hline Vegetables & 295 & 2700 & - & $200(\mathrm{~g}) \dagger \dagger$ \\
\hline Fish & 23 & 640 & - & $34(\mathrm{~g})$ 㧊 \\
\hline
\end{tabular}

en\%, Energy percentage; -, no value set.

* Weighed for the total number of recipients per food bank.

† Dutch nutritional guidelines for a healthy diet ${ }^{(21-23)}$.

‡ Estimated average requirement for men and women in the age ranges 19-30, $31-50$ and $51-70$ years.

§ RDA for men and women in the age ranges $19-30,31-50$ and $51-70$ years.

॥ Daily adequate intake for men and women with normal weight, overweight or undesirable weight gain in the age ranges $19-30,31-50$ and $51-70$ years. The daily adequate intake for overweight people or people with undesirable weight gain is $20-30 / 35$ en\%

If Daily tolerable upper intake level for men and women in the age ranges 19-30, $31-50$ and $51-70$ years.

** Daily tolerable upper intake level for men and women in the age ranges 19-30, $31-50$ and $51-70$ years; daily adequate intake $=$ as low as possible.

†† Daily recommendation for men and women in the age ranges 19-30, 31-50 and $51-70$ years.

¥ Based on the guideline of two times fish per week and the average weight of $120 \mathrm{~g}$ for a single portion.
(SD 11 146) kcal), fruits ranged from 38 (SD 27) to 582 (SD 61) g and vegetables ranged from 187 (SD 66) to 836 (SD 432) g.

The presence of individual food groups in the food parcels and the median provided amount and IQR per product group for a single-person food parcel for one day are shown in Table 2. The following food groups were present in $\geq 75 \%$ of the food parcels: bread, non-alcoholic beverages and vegetables (all 100\%); pastry and cookies (96\%); nuts, seeds and snacks (95\%); sugar, candy, sweet filling and sweet sauces (90\%); milk and milk products (82\%); fats, oils and savoury sauces (78\%); fruits (76\%); and meat, meat products and poultry (75\%), whereas food groups such as legumes (8\%), savoury fillings, for example, peanut butter (17\%), eggs (21\%), soya and vegetarian products (25\%), and fish (27\%) were provided in very few food parcels.

The median provided amount in all ninety-six scored food parcels was highest for the following five food groups - nonalcoholic beverages (437 (IQR 203-850) g), milk and milk products (358 (IQR 91-724) g), bread (256 (IQR 156-398) g), vegetables (247 (IQR 168-455)g) and potatoes (143 (IQR $0-245) \mathrm{g}$ ) - and lowest for the following five food groups - fish (0 (IQR 0-22) g), soya and vegetarian products (0 (IQR 0-1) g), eggs, savoury filling and legumes (all 0 (IQR 0-0)g).

\section{Study population}

In total, 251 Dutch food bank recipients from eleven food banks filled out the questionnaire (Table 3 ). The mean age of the total study sample was 46.3 (sD 10.6) years, and most of the

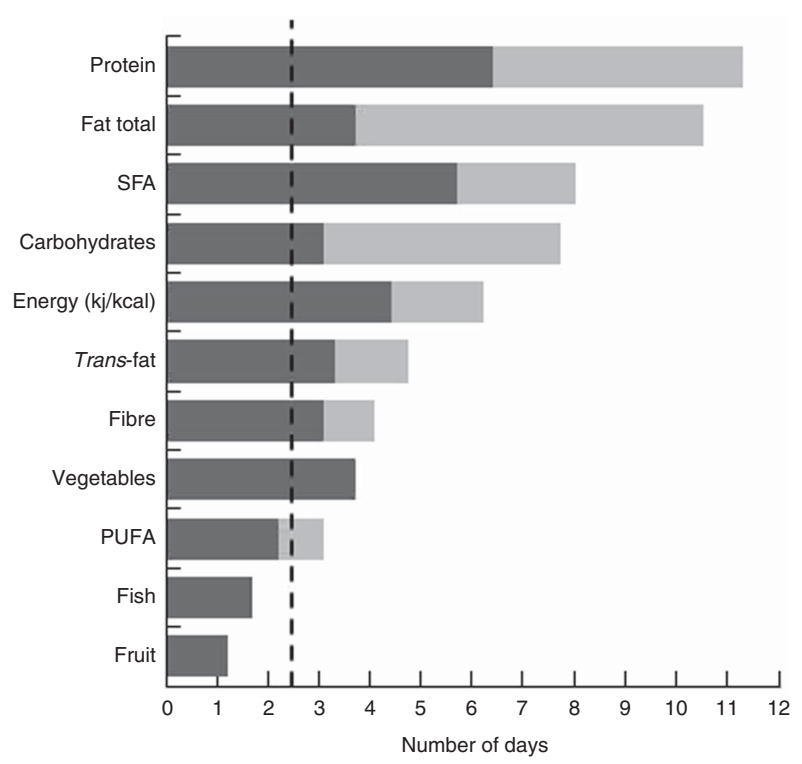

Fig. 1. Number of days the total average weighed absolute amounts of macronutrients, fruits, vegetables and fish (g) from ninety-six food parcels from eleven food banks throughout the Netherlands are provided for in a singleperson food parcel, based on the Dutch nutritional guidelines ${ }^{(21-23)}$. The minimum number of days the absolute amount of nutrients was provided for was based on the lowest recommended values. The maximum number of days the absolute amount of nutrients was provided for was based on the highest recommended values. The reference line is set at $2.5 \mathrm{~d}$. $\square$, Minimum number of days; $\square$, maximum number of days. 
Table 2. The presence of individual food groups in the ninety-six food parcels from eleven food banks throughout the Netherlands and the provided amount per product group for a single-person food parcel for one day (Numbers and percentages; medians and interquartile ranges (IQR))

\begin{tabular}{|c|c|c|c|c|c|c|}
\hline \multirow[b]{2}{*}{ Food groups* } & \multirow[b]{2}{*}{$\%$} & \multirow[b]{2}{*}{$n$} & \multicolumn{2}{|c|}{$\begin{array}{l}\text { Based on all } 96 \\
\text { parcels }(\mathrm{g})\end{array}$} & \multicolumn{2}{|c|}{$\begin{array}{l}\text { Based on parcels containing } \\
\text { foods from specific food group }(\mathrm{g})\end{array}$} \\
\hline & & & Median & IQR & Median & IQR \\
\hline Bread & 100 & 96 & 256 & 156-398 & 256 & 156-398 \\
\hline Non-alcoholic beverages & 100 & 96 & 437 & 203-850 & 437 & 203-850 \\
\hline Vegetables & 100 & 96 & 247 & 168-455 & 247 & $168-455$ \\
\hline Pastry and cookies & 96 & 92 & 93 & $40-1700$ & 100 & $45-171$ \\
\hline Nuts, seeds and snacks & 95 & 91 & 81 & $40-153$ & 87 & $48-157$ \\
\hline Sugar, candy, sweet filling and sweet sauces & 90 & 86 & 61 & $27-129$ & 68 & 40-139 \\
\hline Milk and milk products & 82 & 79 & 358 & $91-724$ & 445 & $200-770$ \\
\hline Fats, oils and savoury sauces & 78 & 75 & 25 & $1-66$ & 46 & 15-83 \\
\hline Fruits & 76 & 73 & 79 & $7-176$ & 127 & $61-232$ \\
\hline Meat, meat products and poultry & 75 & 72 & 124 & $6-228$ & 173 & $101-284$ \\
\hline Grains, flour and rice & 69 & 66 & 50 & $0-137$ & 110 & $46-177$ \\
\hline Potatoes & 69 & 66 & 143 & $0-245$ & 200 & $131-348$ \\
\hline Combined dish & 55 & 53 & 33 & $0-109$ & 86 & 55-195 \\
\hline Cheese & 52 & 50 & 3 & $0-43$ & 40 & $11-87$ \\
\hline Soup & 34 & 33 & 0 & $0-87$ & 150 & $80-216$ \\
\hline Fish & 27 & 26 & 0 & $0-22$ & 59 & $44-77$ \\
\hline Soya and vegetarian products & 25 & 24 & 0 & $0-1$ & 59 & $16-153$ \\
\hline Eggs & 21 & 20 & 0 & $0-0$ & 43 & $26-80$ \\
\hline Savoury filling & 17 & 16 & 0 & $0-0$ & 50 & $36-85$ \\
\hline Legumes & 8 & 8 & 0 & $0-0$ & 139 & 74-252 \\
\hline
\end{tabular}

* Food groups are based on the Dutch Food Composition Table ${ }^{(27)}$.

participants were recipients of the food bank $>12$ months. The majority of participants $(52.4 \%)$ had a medium level of education. Of the participants, $56 \cdot 8 \%$ were either overweight or obese. Furthermore, $72.9 \%$ of the participants were food insecure, of which $40 \cdot 4 \%$ had very low food security. A detailed description of the participants' characteristics have been described elsewhere ${ }^{(2)}$. Most of the participants were completely responsible for the daily grocery shopping (59.6\%), (very) satisfied with the contents of the food parcel (75.7\%) and in general perceived the contents of the food parcel as (very) healthy (61.8\%). Of the participants, 39.4\% used all foods provided, $22.7 \%$ never used perishable foods and $13.5 \%$ never used non-perishable foods beyond their expiration date.

Food bank recipients reported that they most often buy foods from the following five food groups to supplement the food parcel: potatoes $(18.6 \%)$, cheese $(12.8 \%)$, meat, meat products and poultry $(12.8 \%)$, fruits $(9.5 \%)$, and milk and milk products $(9.5 \%)$, whereas vegetables $(6.4 \%)$ and fish (4.6\%) were bought less often. The main reported reason for not using foods from the food parcel was the presence of foods beyond the expiration date (45.9\%), followed by not liking the food (22.3\%) and other reasons (19.6\%) such as religion or allergy.

\section{Discussion}

Our study shows that the nutritional content of food parcels provided by Dutch food banks was not in line with the Dutch nutritional guidelines ${ }^{(21-23)}$. The food parcels provided very high amounts of energy, protein and SFA, whereas the provided amounts of fruits and fish were very low. Furthermore, food bank recipients seldom bought fruits and fish to supplement their food parcel.

Although food banks across the world differ in the way they collect and provide foods, the few available studies from Canada and the USA showed similar results as us regarding the wide range in availability of macronutrients and food groups ${ }^{(30-32)}$ and the low availability of fruits ${ }^{(31-33)}$. A study by Jessri et al. ${ }^{(34)}$ showed that none of the food hampers (i.e. one- to five-person hampers) met the dietary reference intake recommendations for vitamin A and $\mathrm{Zn}$, and that nutrients missing from the food hampers could be obtained from fresh fruits, vegetables, dairy products and meat products. Teron \& Tarasuk ${ }^{(30)}$ found that food parcels contained more energy and protein than what is recommended for the number of days that the food parcel was intended for. In contrast, Irwin et al. ${ }^{(31)}$ found that food parcels contained lower amounts of energy than recommended. In general, the provided amounts of fruit in the food parcels were relatively low and below dietary recommendations ${ }^{(31-33)}$.

Food parcels that are not in line with dietary recommendations may lead to unhealthy nutritional intake, which may consequently lead to a higher risk of nutrition-related chronic diseases. Studies on the nutritional intake of food bank recipients are, however, scarce. A study by Leung et al. ${ }^{(35)}$ shows that adults participating in a Supplemental Nutrition Assistance Program (SNAP) had diets poorer in quality than non-SNAP participants, who also did not meet the recommended guidelines (e.g. for fruits, vegetables and fish). In addition, poor consumption frequencies of especially fruits, vegetables and dairy products were observed in French food aid users ${ }^{(36)}$. 
Table 3. Characteristics of the 251 Dutch food bank recipients from eleven food banks throughout the Netherlands

(Mean value and standard deviation; numbers and percentages; numbers and ranges; $n$ 251)*

\begin{tabular}{|c|c|c|}
\hline Characteristics & $n$ & $\%$ \\
\hline \multicolumn{3}{|l|}{ Age (years) } \\
\hline Mean & \multicolumn{2}{|c|}{$46 \cdot 3$} \\
\hline SD & \multicolumn{2}{|c|}{$10 \cdot 6$} \\
\hline \multicolumn{3}{|l|}{ Sex } \\
\hline Male & 93 & 37.1 \\
\hline Female & 158 & $62 \cdot 9$ \\
\hline \multicolumn{3}{|l|}{ Duration of being recipient } \\
\hline 0-6 months & 91 & $36 \cdot 3$ \\
\hline $6-12$ months & 63 & $25 \cdot 1$ \\
\hline$>12$ months & 97 & 38.6 \\
\hline \multicolumn{3}{|l|}{ Household size } \\
\hline 1 person & 102 & 40.6 \\
\hline 2-4 persons & 108 & 43.0 \\
\hline$\geq 5$ persons & 41 & $16 \cdot 3$ \\
\hline \multicolumn{3}{|l|}{ Educational level } \\
\hline Low & 34 & 13.6 \\
\hline Medium & 131 & $52 \cdot 4$ \\
\hline High & 85 & 34.0 \\
\hline \multicolumn{3}{|l|}{ Weight status } \\
\hline Underweight $\left(\mathrm{BMl}<18.5 \mathrm{~kg} / \mathrm{m}^{2}\right)$ & 8 & 3.3 \\
\hline Normal weight (BMl $18-24.9 \mathrm{~kg} / \mathrm{m}^{2}$ ) & 98 & $40 \cdot 0$ \\
\hline Overweight (BMI 25-29.9 kg/ $\mathrm{m}^{2}$ ) & 70 & 28.6 \\
\hline Obese $\left(\mathrm{BMI} \geq 30 \mathrm{~kg} / \mathrm{m}^{2}\right)$ & 69 & $28 \cdot 2$ \\
\hline \multicolumn{3}{|l|}{$\begin{array}{l}\text { Money spent on groceries per person per } \\
\text { week in euros }\end{array}$} \\
\hline Mean & & \\
\hline Ranges & & \\
\hline \multicolumn{3}{|l|}{ Responsibility daily grocery shopping } \\
\hline Not at all & 3 & $1 \cdot 2$ \\
\hline A small part & 27 & $10 \cdot 8$ \\
\hline Half & 30 & $12 \cdot 0$ \\
\hline A large part & 41 & $16 \cdot 4$ \\
\hline Completely & 149 & 59.6 \\
\hline \multicolumn{3}{|l|}{ Satisfaction with food parcel } \\
\hline Very unsatisfied & 4 & 1.6 \\
\hline Unsatisfied & 11 & 4.4 \\
\hline Not unsatisfied/not satisfied & 46 & $18 \cdot 3$ \\
\hline Satisfied & 140 & 55.8 \\
\hline Very satisfied & 50 & 19.9 \\
\hline \multicolumn{3}{|c|}{ Perception healthiness contents of food parcel } \\
\hline Very unhealthy & 0 & 0 \\
\hline Unhealthy & 18 & $7 \cdot 2$ \\
\hline Not unhealthy/not healthy & 77 & 30.9 \\
\hline Healthy & 140 & $56 \cdot 2$ \\
\hline Very healthy & 14 & 5.6 \\
\hline \multicolumn{3}{|l|}{ Use of all products from food parcel } \\
\hline Never & 9 & 3.6 \\
\hline Sometimes & 143 & 57.0 \\
\hline Always & 99 & 39.4 \\
\hline \multicolumn{3}{|c|}{ Use of perishable foods beyond expiration date } \\
\hline Never & 57 & $22 \cdot 7$ \\
\hline Sometimes & 154 & 61.4 \\
\hline Always & 40 & $15 \cdot 9$ \\
\hline \multicolumn{3}{|l|}{$\begin{array}{l}\text { Use of non-perishable foods beyond } \\
\text { expiration date }\end{array}$} \\
\hline Never & 34 & 13.5 \\
\hline Sometimes & 159 & $62 \cdot 9$ \\
\hline Always & 59 & 23.5 \\
\hline \multicolumn{3}{|l|}{ Level of food security } \\
\hline Food secure & 68 & $27 \cdot 2$ \\
\hline Low food secure & 109 & 43.4 \\
\hline Very low food secure & 74 & 29.5 \\
\hline
\end{tabular}

* For age, educational level and responsibility of grocery shopping $n$ was 250; for perception healthiness contents of food parcel $n$ was 249; for weight status and money spent on groceries in euros per person per week $n$ was 245 .
In addition, $33 \%$ of the participants in this study had low serum $\beta$-carotene concentrations, $26.5 \%$ were at moderate risk of vitamin $\mathrm{C}$ deficiency, almost $70 \%$ were at risk of folate deficiency and $85.6 \%$ were at risk of vitamin D deficiency ${ }^{(36)}$. Robaina \& Martin ${ }^{(6)}$ showed that food pantry users consumed limited amounts of fruits, vegetable and fibre. In addition, Depa et al. ${ }^{(37)}$ showed considerably lower fruit intakes in disadvantaged people using food banks compared with the low SES populations of national surveys. Only one study showed that the nutritional intake of food bank users was not worse compared with the general population ${ }^{(38)}$.

Overall, in our study, the nutritional content of a singleperson food parcel for one day was comparable with the dietary intake of the general Dutch population ${ }^{(39)}$. However, the amounts of energy, fibre, vegetables and fish were more than 2-fold higher compared with the dietary intake of the general population. It is important to take into account that we compared the contents of the food parcels with dietary intake of the general Dutch population, although we do not know what is actually eaten from the food parcel.

Our study seems to show some conflicting results. The amounts of energy and macronutrients such as proteins and carbohydrates in the food parcel were provided for more than $2.5 \mathrm{~d}$ and $57 \%$ of the participants was either overweight or obese. However, $73 \%$ of our study population was food insecure and only 39\% always used all foods provided. The main reported reason for not using foods from the food parcel was the presence of food beyond the expiration date (45.9\%), followed by not liking the food $(22 \cdot 3 \%)$ and other reasons (19.6\%) such as religion or allergy. In addition, fruit was provided in $76 \%$ and fish in $27 \%$ of the food parcels, and the amounts of fruits and fish in a single-person food parcel for a day were below the recommendation. In spite of that, only $10 \%$ of the recipients bought fruits and $5 \%$ fish to supplement the food parcel. Furthermore, foods from the food groups bread, non-alcoholic beverages and vegetables were provided in all food parcels, whereas foods from protein-containing food groups such as fish, soya and vegetarian products, eggs and legumes were provided in very few food parcels. However, the majority of food bank recipients were (very) satisfied with the contents of the food parcel and in general perceived the contents of the food parcels as (very) healthy.

The results of our study should be interpreted in the context of its strengths and limitations. Strengths include the methods that we used to analyse the nutritional quality, because it takes the variability between food parcels, types of food parcels scored per food bank and the total number of recipients per participating food bank into account. For example, if smaller food banks provided high amounts of vegetables, whereas larger food banks provided low amounts of vegetables, the overall mean weighed for the total number of recipients per food bank would not be overestimated. Therefore, our results are representative of an average food parcel from an average food bank in the Netherlands.

For the current study, we compared the nutritional content of the food parcels with the Dutch nutritional guidelines for a healthy diet. Several issues should be addressed. First, we measured the contents of the food parcels but not the actual 
food intake. Food bank recipients did not eat certain foods provided because of personal preferences or poor quality of the foods. Second, we did not measure foods bought or foods provided by others. Third, in November 2015, new guidelines for a healthy diet were published in the Netherlands ${ }^{(40)}$, in which the fish guideline changed from 2 portions/week to 1 portion/week. Hereby, the recommendation decreased from $34 \mathrm{~g} / \mathrm{d}$ to $17 \mathrm{~g} / \mathrm{d}$, and food parcels would consequently meet the new fish guideline. However, we would like to stress that this does not necessarily mean that fish consumption also met the fish guideline. Fourth, the contents of the food parcel were intended for $2.5 \mathrm{~d}$ but some foods provided were intended to be used for more than $2.5 \mathrm{~d}$ (e.g. a jar of peanut butter, a bag of sugar), which may have led to an overestimation of the availability of some macronutrients. Unfortunately it was not possible to mark foods that were used as kitchen pantry stock. The usual classification of foods as stock foods may not be applicable to our study population. Food bank recipients largely depend on the contents of food parcels, and therefore often finish foods that are intended for more than $2.5 \mathrm{~d}$ faster. For example, a jar of jam may be finished much faster if there are no alternatives to choose from. In future research, it is important to collect dietary intake data of Dutch food bank recipients to verify whether the definition of stock foods is applicable to our study population and what is actually eaten from the food parcel.

Furthermore, from the literature it is known that seasonal variation may influence the type and/or quantity of fruit and vegetable intake of adults ${ }^{(41)}$. Owing to the broad period of data collection - October 2010 to April 2011 - seasonal variation in fruit and vegetable supply may have led to bias.

Finally, weight status was based on self-reported height and weight, and therefore may have been biased. A study by Ver Ploeg et al. ${ }^{(42)}$ reported that overweight women who received food stamp benefits were less likely to recognise that they were overweight than eligible non-participants.

Our study shows that the nutritional quality of food parcels supplied by Dutch food banks was not in line with the nutritional guidelines. As food bank recipients largely rely on the contents of food parcels, the current contents are not likely to contribute to a healthy diet. This is a serious issue because of the high prevalence of food insecurity and overweight in this population, and consequently high risks of nutrition-related chronic diseases. Our results highlight the need to improve the nutritional quality of the food parcels provided by Dutch food banks. Furthermore, efforts may be needed not just to change the contents of the food parcels but also to change consumer demand for some of the food items through, for example, skill building, nutrition education and tasting.

In the present concept of the food banks, food parcels could be improved by providing handles for composing a food parcel according to the nutritional guidelines for a healthy diet as much as possible. Moreover, information on foods that are necessary and desirable for composing a healthier food parcel could be given to donating companies, as well as companies who potentially could donate food. Nevertheless, more research is needed on the nutritional intake of food bank recipients, whether providing handles for composing a food parcel according to the nutritional guidelines for a healthy diet improves the nutritional content of the food parcels and how this improvement affects the nutritional intake.

\section{Acknowledgements}

The authors thank all food banks that participated for their cooperation, all food bank recipients for their participation and all research assistants and MSc and BSc interns for their help in collecting data.

The Food Bank Study was funded by a grant from the Netherlands Organization for Health Research and Development (115100003).

J. E. N., I. A. B. and M. V. designed the research. J. E. N. and S. C. D. conducted the research. J. E. N. performed the complete data analyses, writing of the first draft of the manuscript and revision of the manuscript. S. C. D., M. V. and I. A. B. provided significant advice concerning interpretation of the results and critical review of the manuscript. J. E. N. had primary responsibility for the final content. All the authors were involved in the development of the manuscript and approved the final version.

The authors declare that there are no conflicts of interest.

\section{Supplementary material}

For supplementary material/s referred to in this article, please visit http://dx.doi.org/doi:10.1017/S0007114516002087

\section{References}

1. Voedselbanken Nederland (the Dutch Food Bank) (2014) Feiten en Cijfers Voedselbanken Nederland (Facts and Figures of the Dutch Food Bank). http://www.voedselbankennederland.nl/ (accessed September 2015).

2. Neter JE, Dijkstra SC, Visser M, et al. (2014) Food insecurity among Dutch food bank recipients: a cross-sectional study. BMJ Open 4, e004657.

3. Anderson S (1990) Core indicators of nutritional state for difficult-to-sample populations. J Nutr 120, 1559-1600.

4. Duffy P, Zizza C, Jacoby J, et al. (2009) Diet quality is low among female food pantry clients in Eastern Alabama. J Nutr Educ Behav 41, 414-419.

5. Kendall A, Olson CM \& Frongillo EA (1996) Relationship of hunger and food insecurity to food availability and consumption. J Am Diet Assoc 96, 1019-1024; quiz 1025-1016.

6. Robaina KA \& Martin KS (2013) Food insecurity, poor diet quality, and obesity among food pantry participants in Hartford, CT. J Nutr Educ Behav 45, 159-164.

7. Tarasuk VS (2001) Household food insecurity with hunger is associated with women's food intakes, health and household circumstances. J Nutr 131, 2670-2676.

8. Lee SE, Song YJ, Kim Y, et al. (2016) Household food insufficiency is associated with dietary intake in Korean adults. Public Health Nutr 19, 1112-1121.

9. Hanson KL \& Connor LM (2014) Food insecurity and dietary quality in US adults and children: a systematic review. Am J Clin Nutr 100, 684-692.

10. Dixon LB, Winkleby MA \& Radimer KL (2001) Dietary intakes and serum nutrients differ between adults from food-insufficient and food-sufficient families: Third National Health and Nutrition Examination Survey, 1988-1994. J Nutr 131, 1232-1246. 
11. Kirkpatrick SI \& Tarasuk V (2008) Food insecurity is associated with nutrient inadequacies among Canadian adults and adolescents. J Nutr 138, 604-612.

12. Rose D \& Oliveira V (1997) Nutrient intakes of individuals from food-insufficient households in the United States. Am J Public Health 87, 1956-1961.

13. Bell M, Wilbur L \& Smith C (1998) Nutritional status of persons using a local emergency food system program in middle America. I Am Diet Assoc 98, 1031-1033.

14. Muirhead V, Quinonez C, Figueiredo R, et al. (2009) Oral health disparities and food insecurity in working poor Canadians. Community Dent Oral Epidemiol 37, 294-304.

15. Che J \& Chen J (2001) Food insecurity in Canadian households. Health Rep 12, 11-22.

16. Hampton T (2007) Food insecurity harms health, well-being of millions in the United States. JAMA 298, 1851-1853.

17. Holben DH \& Pheley AM (2006) Diabetes risk and obesity in food-insecure households in rural Appalachian Ohio. Prev Chronic Dis 3, A82.

18. Laraia BA (2013) Food insecurity and chronic disease. Adv Nutr 4, 203-212.

19. Seligman HK, Laraia BA \& Kushel MB (2010) Food insecurity is associated with chronic disease among low-income NHANES participants. J Nutr 140, 304-310.

20. Townsend MS, Peerson J, Love B, et al. (2001) Food insecurity is positively related to overweight in women. $J$ Nutr $\mathbf{1 3 1}$, $1738-1745$.

21. Gezondheidsraad (Health Council of the Netherlands) (2006) Richtlijnen goede voeding 2006 - achtergronddocument (Guidelines for a healthy diet 2006 - background document). Publicatie no. (Publication no.) A06/08. Den Haag: Gezondheidsraad.

22. Health Council of the Netherlands (2001) Dietary Reference Intakes: Energy, Proteins, Fats and Digestible Carbohydrates, Publication no. 2001/19R (corrected edition: June 2002). The Hague: Health Council of the Netherlands.

23. Health Council of the Netherlands (2006) Guideline for Dietary Fiber Intake, Publication no. 2006/03. The Hague: Health Council of the Netherlands.

24. Rijksinstituut voor Volksgezondheid en Milieu (National Institute for Public Health and the Environment) (2010) NEVO-tabel (Dutch Food Composition Table). Nederlands Voedingsstoffenbestand 2010 (Dutch Food Composition Database 2010). Den Haag: RIVM.

25. Donders-Engelen M, Heijden van der L \& Hulshof K (2003) Maten, Gewichten en Codenummers 2003 (Measures, Weights and Codes 2003). Wageningen: Gezamenlijke produktie van Afdeling Humane Voeding, Wageningen Universiteit en TNO Voeding te Zeist.

26. World Health Organization (WHO) Expert Committee (1995) Physical Status: The Use and Interpretation of Anthropometry. Technical Report Series. Geneva: WHO.

27. Voedingscentrum (The Netherlands Nutrition Centre) (2001) NEVO-tabel: Nederlands Voedingsstoffenbestand 2001/ Stichting Nederlands Voedingsstoffenbestand (Dutch Food
Composition Table: Dutch Food Composition Database 2001/ Dutch Food Composition Database Foundation). Den Haag: Voedingscentrum.

28. Bickel G, Nord M, Price C, et al. (2000) Guide to Measuring Household Food Security, Revised 2000. Washington, DC: US Department of Agriculture.

29. Blumberg SJ, Bialostosky K, Hamilton WL, et al. (1999) The effectiveness of a short form of the Household Food Security Scale. Am J Public Health 89, 1231-1234.

30. Teron AC \& Tarasuk VS (1999) Charitable food assistance: what are food bank users receiving? Can J Public Health 90 , 382-384.

31. Irwin JD, Ng VK, Rush TJ, et al. (2007) Can food banks sustain nutrient requirements? A case study in Southwestern Ontario. Can J Public Health 98, 17-20.

32. Akobundu UO, Cohen NL, Laus MJ, et al. (2004) Vitamins A and $\mathrm{C}$, calcium, fruit, and dairy products are limited in food pantries. J Am Diet Assoc 104, 811-813.

33. Hoisington A, Manore MM \& Raab C (2011) Nutritional quality of emergency foods. J Am Diet Assoc 111, 573-576.

34. Jessri M, Abedi A, Wong A, et al. (2014) Nutritional quality and price of food hampers distributed by a campus food bank: a Canadian experience. J Health Popul Nutr 32, 287-300.

35. Leung CW, Ding EL, Catalano PJ, et al. (2012) Dietary intake and dietary quality of low-income adults in the Supplemental Nutrition Assistance Program. Am J Clin Nutr 96, 977-988.

36. Castetbon K, Mejean C, Deschamps V, et al. (2011) Dietary behaviour and nutritional status in underprivileged people using food aid (ABENA study, 2004-2005). J Hum Nutr Diet 24, 560-571.

37. Depa J, Hilzendegen C, Tinnemann P, et al. (2015) An explorative cross-sectional study examining self-reported health and nutritional status of disadvantaged people using food banks in Germany. Int J Equity Health 14, 141.

38. Jacobs Starkey L, Gray-Donald K \& Kuhnlein HV (1999) Nutrient intake of food bank users is related to frequency of food bank use, household size, smoking, education and country of birth. J Nutr 129, 883-889.

39. Rossum van C, Fransen H, Verkaik-Kloosterman J, et al. (2011) Dutch National Food Consumption Survey 2007-2010. Diet of Children and Adults Aged 7 to 69 Years. Bilthoven: National Institute for Public Health and the Environment.

40. Health Council of the Netherlands (2015) Dutch Dietary Guidelines 2015, Publication no. 2015/24E. The Hague: Health Council of the Netherlands.

41. Schatzer M, Rust P \& Elmadfa I (2010) Fruit and vegetable intake in Austrian adults: intake frequency, serving sizes, reasons for and barriers to consumption, and potential for increasing consumption. Public Health Nutr $\mathbf{1 3}$, 480-487.

42. Ver Ploeg ML, Chang HH \& Lin BH (2008) Over, under, or about right: misperceptions of body weight among food stamp participants. Obesity (Silver Spring) 16, 2120-2125. 